\title{
The effect of prolonged visual deprivation on various cutaneous and auditory measures'
}

\section{J. PHELPS and JOHN P. ZUBEK, University of Manitoba, Winnipeg, Canada}

Subjects who were placed in darkness for a week but otherwise were exposed to a normal and varied sensory environment showed a significant increase in absolute pressure sensitivity of the finger, forearm, neck, and leg, but not of the palm, relative to a group of controls. Measures of tactual and auditory localization, on the other hand, showed no significant changes after visual deprivation. These results provide some experimental support for Schultz's (1965) sensoristatic theory.

Three studies (Duda \& Zubek, 1965; Zubek et al, 1964a, b) performed recently at the University of Manitoba have reported a significant increase in tactual acuity and in heat and pain sensitivity after a week of visual deprivation. A significant improvement in auditory discrimination (auditory flutter fusion frequency) but not of the absolute threshold of hearing for pure tones was also reported. The purpose of this experiment was to employ a variety of additional cutaneous and auditory measures in order to determine how general or limited these intersensory facilitatory effects may be.

\section{METHOD}

Sixteen male university students, each wearing a black mask, were placed in groups of two in an ordinary room for a prescribed period of one week. Apart from the exposure to constant darkness, their environment was quite normal. No gloves were worn and no restrictions were placed on their motor activity or on conversation with one another. Furthermore, a radio was available in the room at all times. All 16 Ss successfully endured the week of darkness, but the data from one $S$ was rejected because of his failure to adhere to the experimental procedure.

Two tactual (pressure sensitivity and point localization) and two auditory measures (absolute and differential sound localization) were administered before and at the end of one week of visual deprivation and, subsequently, at intervals of 1 , 2 , and 5 days after the termination of the experimental condition. A practice session, for purposes of test familiarization, was given a day prior to the experiment. The details of the four tests are as follows.

\section{Pressure Sensitivity}

The absolute pressure sensitivity of the skin was determined for the middle of the index finger, palm, volar surface of the forearm $(8 \mathrm{~cm}$ below the elbow), back of the neck, and front of the leg $(15 \mathrm{~cm}$ below the knee). The determinations were made by the Semmes-Weinstein pressure aesthesiometer (Shaw Laboratories, New York) which consists of a series of 20 nylon monofilaments, $38 \mathrm{~mm}$ in length and ranging in diameter from 0.06 to $1.14 \mathrm{~mm}$. Each hair is calibrated in terms of the logarithm of the force (in milligrams) required to bend it maximally. The procedure used was similar to that employed by Semmes et al (1960) with one modification, viz., the employment of a mechanical rather than a manual presentation of the stimuli. The filaments were placed in a metal arm which was lowered automatically toward the skin, at a constant speed, until the filament was bent maximally. The mechanical arm was subsequently withdrawn, again at a constant speed. Each filament was applied for approximately $3 \mathrm{sec}$ with intervals of 10 to $15 \mathrm{sec}$ between individual applications. The method of limits was employed to determine the thresholds. With the exception of the back of the neck which received eight trials, four trials (ADAD) were given on each finger, palm, forearm, and leg. Prior to testing, all areas with hair were shaved. The order of testing was as follows: left finger, left palm, left forearm, neck, right finger, right palm, right forearm, left leg, and right leg. In determining the threshold, the results on the two homologous skin areas, e.g., left and right palm, were combined.

\section{Tactual Localization}

The error of localization of a single point applied to the volar surface of each forearm was determined for the proximo-distal axis. $\mathbf{S}$ was seated, blindfolded, with his arm on a pad while five points, each $5 \mathrm{~mm}$ apart, were marked lengthwise down the center of his forearm. Each of the five points was stimulated twice, in a random order, with the single point of a two-point aesthesiometer. The stimulus was applied for $1 \mathrm{sec}$ after which $\mathrm{S}$, using the tip of a sharp pencil, indicated the apparent location of the stimulus. The measure recorded was the distance, in millimeters, from the point stimulated to the tip of S's pencil. The error of localization was the mean of 20 trials, 10 on each forearm.

\section{Absolute Auditory Localization}

Auditory localization was measured by means of a sound cage (Marietta Apparatus Co.) consisting of a chair with an adjustable head rest and a high quality miniature speaker, attached to a moveable arm, which produced a low-volume click when a button was pressed. The sound cage was placed in the center of a sound-proofed room measuring $10 \times 15 \mathrm{ft}$. S, seated in a chair and wearing a black mask, was required to keep his head in the same position throughout the test and his eyes directed straight ahead. The method of average error was used to measure the ability to localize the click at either 30 or $60 \mathrm{deg}$ to the right of center in a horizontal plane in front of S and at the level of the ears. The error was the difference in degrees between the location of the sound source and the location of the index finger of the extended right arm, placed at a position approximately $1 \mathrm{in}$. below where the speaker was believed to be. Ten trials were administered: four at $30 \mathrm{deg}$, four at $60 \mathrm{deg}$, and two "check" trials directly in front of S. The trials were so arranged that the same location was never tested twice in a row and the choice of which stimulus position was to be presented first on a test day was determined on a random basis.

\section{Differential Auditory Localization}

The sound cage, described previously, was also used to measure differential auditory localization. The method of limits was employed to determine the just perceptible difference in location of the auditory stimulus when the standard was presented at either $30 \mathrm{deg}$ or $60 \mathrm{deg}$ to the right of center in the horizontal plane. Each judgment resulted from two clicks, separated by 3 sec. The first was the standard, presented at either 30 or $60 \mathrm{deg}$ to the right of center, and the second was the comparison stimulus to be judged as being either in the "same" place or to the "right" of the first. Two blocks of trials were administered: one for the 30-deg and the other for the $60-\mathrm{deg}$ determination. Each block consisted of four descending and four ascending trials. Beginning well above (descending) or well below (ascending) the expected just perceptible difference, the angular distance between the standard and comparison stimulus was made either smaller or larger in steps of $1 \mathrm{deg}$. The setting recorded was the first time $S$ said "right" in the ascending series and the last time he said "right" in the descending series. Periodic "check" trials were used in which the two clicks were presented at the standard position only. 
A group of 15 control Ss (male undergraduate students) received the same tests, in the same order, and at the same time intervals as the experimentals.

\section{RESULTS}

Two-tailed t-tests for independent measures were performed on the pre-post difference scores of the experimental and control Ss. The results revealed a heightened sensitivity to pressure on all skin areas, in the experimental relative to the control Ss, at the end of the one-week period of visual deprivation. Statistically significant results were obtained on the finger $(\mathrm{p}<.01)$, forearm $(\mathrm{p}<.002)$, neck $(\mathrm{p}<.05)$, and leg $(p<.05)$, but not on the palm. Furthermore, significant aftereffects were still present on postdeprivation Day 2 for the finger $(p<.01)$ and on postdeprivation Day 5 for the forearm $(\mathrm{p}<.02)$ and neck $(\mathrm{p}<.05)$.

The results on the remaining three measures, i.e., tactual localization and absolute and differential auditory localization, showed no significant differences between the experimental and control Ss. Furthermore, no indication of a trend toward increased accuracy of localization in the experimental Ss was evident on any of the measures.

\section{DISCUSSION}

The results of this experiment have indicated that prolonged visual deprivation can produce a significant increase in pressure sensitivity of the finger, forearm, neck, and leg, with the aftereffects persisting for several days after the restoration of normal visual stimulation. A trend toward increased sensitivity of the palm was also observed but the change was not statistically significant. Essentially similar results, derived from other cutaneous measures, have been demonstrated by Zubek et al (1964a) who reported that a week of darkness produced not only a significant increase in heat and pain sensitivity of the forearm but also an increase in tactual acuity of the index finger, palm, and forearm. Furthermore, the effects persisted for a number of days after the termination of the experimental condition. In view of this general increase in cutaneous sensitivity, as indicated by a variety of measures, it is surprising that tactual localization did not show a significant improvement in accuracy or even a trend toward improvement. One possible explanation of this negative finding may be that tactual localization is much more dependent on practice and learning than are the other cutaneous measures which have been employed (Halman \& Wright, 1960). A similar explanation may account for the nonsignificant results on both absolute and differential auditory localization. Held (1955), for example, has shown that experience in localizing a sound for a period of $1 \mathrm{~h}$ produced a 10-deg change in the accuracy of localization.

Since practice may be the main factor responsible for producing these negative results, it might be fruitful, in future research, to employ a variety of other auditory measures which are only minimally dependent upon practice and learning, e.g., frequency and intensity discrimination. If this were to be done, the results might indicate that prolonged visual deprivation can produce auditory facilitatory effects of a much more general nature than appears to be the case at present (Duda \& Zubek, 1965), particularly since Gibby
(1967) has recently reported that visual deprivation of only 3-h duration can produce a significant improvement in both pitch and loudness discrimination as measured by the Seashore Test of Musical Talents.

The demonstration of a significant increase in pressure sensitivity of the finger, forearm, neck, and leg but no change on the palm is puzzling especially since Zubek et al (1964a) have reported a significant improvement in tactual acuity of the palm (two-point threshold) after a week of visual deprivation. It is important to note, however, that a similar differential pattern has been observed by Weinstein et al (1967) after a four-day period of tactual occlusion of a circumscribed area of the forearm and palm. Using a von Frey hair technique, Weinstein reported a significant increase in absolute pressure sensitivity of the forearm but no significant change in the sensitivity of the palm. These results, therefore, appear to suggest that a prolonged period of sensory restriction (either visual or tactual) produces an effect on the pressure sensitivity of the palm which is different from that observed on more proximal or distal areas of the arm. The reason for this anomaly must await further research.

In conclusion, these results, indicating an increase in pressure sensitivity after prolonged visual deprivation, are consistent with one of the predictions proposed by Schultz (1965) in his sensoristatic theory of the nervous system, i.e., "when stimulus variation is restricted, central regulation of threshold sensitivities will function to lower sensory thresholds. Thus, the organism becomes increasingly sensitized to stimulation in an attempt to restore the balance [p. 32]." Furthermore, according to this theory, an improvement in tactual and auditory localization might not be expected since these performance measures largely involve learning rather than threshold determinations of sensitivity.

\section{REFERENCES}

DUDÁ, P., \& ZUBEK, J. P. Auditory sensitivity after prolonged visual deprivation. Psychonomic Science, 1965, 3, 359-360.

GIBBY, R. G. The effects of visual restriction on visual and auditory discrimination. Dissertation Abstracts, 1967, 27(9-B), 3308.

HALMAN, C. R. E., \& WRIGHT, G. H. Tactile localization. Brain, 1960 , $83,677-700$

HELD, R. Shifts in binaural localization after prolonged exposures to atypical combinations of stimuli. American Journal of Psychology, $1955,69,526-548$.

SCHULTZ, D. P. Sensory restriction. New York: Academic Press, 1965. SEMMES, J., WEINSTEIN, S., GHENT, L., \& TEUBER, H. L. Somatosensory changes after penetrating brain wounds in man. Cambridge: Harvard University. Press, 1960.

WEINSTEIN, S., RICHLIN, M., WEISINGER, M., \& FISHER, L. The effects of sensory deprivation on sensory, perceptual, motor, cognitive, and physiological functions. Technical Report CR-727, March 1967, NASA, Washington, D.C.

ZUBEK, J. P., FLYE, J., \& AFTANAS, M. Cutaneous sensitivity after prolonged visual deprivation. Science, 1964a, 144, 1591-1593.

ZUBEK, J. P., FLYE, J., \& WILLOWS, D. Changes in cutaneous sensitivity after prolonged exposure to unpatterned light. Psychonomic Science, 1964b, 1, 283-284.

\section{NOTE}

1. This research was supported by the Defense Research Board, Canada (No. 9425-08) and by the National Research Council, Canada (APA-290). assumed to provide a good estimate of the true magnitude of effect, then we can use Table 1 to indicate the appropriate total sample size. In the above example with $r_{m}=.50$, the column for $p=.05$ shows that a sample size $=14$, or a total of $16 \mathrm{Ss}$, would be needed to obtain significance at the .05 level. With the original table additional material and procedures are available for estimating sample size.

\section{REFERENCES}

COHEN, J. Some statistical issues in psychological research. In B. B. Wolman (Ed.), Handbook of clinical psychology. New York: McGraw-Hill, 1967. Pp. 95-121.

FRIEDMAN, H. Magnitude of experimental effect and a table for its rapid estimation. Psychological Bulletin, 1968, 70, 245-251. NOTE

1. Supported in part by NASA Research Grant NGR 47-006-028. 